\title{
The first-light APEX submillimeter heterodyne instrument FLASH
}

\author{
S. Heyminck ${ }^{1}$, C. Kasemann ${ }^{1}$, R. Güsten ${ }^{1}$, G. de Lange ${ }^{2}$, and U. U. Graf ${ }^{3}$ \\ 1 Max-Planck-Institut für Radioastronomie, Auf dem Hügel 69, 53121 Bonn, Germany \\ e-mail: heyminck@mpifr-bonn.mpg.de \\ 2 SRON Netherlands Institute for Space Research, Postbus 800, 9700 AV Groningen, The Netherlands \\ 3 I. Physikalisches Institut der Universität zu Köln, Zülpicher Straße 77, 50937 Köln, Germany \\ Received 11 April 2006 / Accepted 16 May 2006
}

ABSTRACT

\begin{abstract}
Development of a dual-color heterodyne instrument for use with the Atacama Pathfinder EXperiment. Commissioning of the APEX began in mid 2004, and regular science operation has been performed since July 2005 . Verification of the telescope required a dualchannel receiver operating at (short) submillimeter wavelengths. It was important for the characterization of the telescope to observe at the highest possible frequency at which routine observations can be performed. For pointing, focus, and tracking verification (simultaneous) operation at lower frequencies was requested. We developed FLASH operating on two channels simultaneously at orthogonal polarizations - in the $460 \mathrm{GHz}$ and $810 \mathrm{GHz}$ atmospheric windows. The system performs with a wide tuning range (420-500 GHz, 780-880 GHz) and intermediate frequency bandwidths of 2 and $4 \mathrm{GHz}$, respectively. As backends, we operate two fast-Fourier transform spectrometers (FFTS) with $2 \times 1 \mathrm{GHz}$ bandwidth each and a maximum of 16384 channels. The receiver has been in continuous operation since June 2004. While first used for the telescope commissioning, since the middle of last year the instrument has served as the high-frequency workhorse on APEX. Simultaneous observations of the rotational transitions of warm carbon monoxide $(J=4-3$ and $J=7-6)$ and of the two fine-structure lines of atomic carbon are scientifically attractive. FLASH is a principal investigator instrument, available to the APEX-user community on a collaborative basis with MPIfR. A state-of-the-art dualchannel heterodyne instrument has been developed, which made timely commissioning of the APEX possible. Most of the scientific results presented in this special issue rely on data derived with FLASH.
\end{abstract}

Key words. instrumentation: miscellaneous - techniques: spectroscopic

\section{Introduction}

For the commissioning of the Atacama Pathfinder EXperiment (APEX ${ }^{1}$, Güsten et al. 2006), which started in mid 2004 and for first-light science, we developed FLASH (first light APEX submillimeter heterodyne instrument). FLASH is a dual-frequency closed-cycle cooled heterodyne MPIfR principal investigator (PI) receiver, operating in the $460 \mathrm{GHz}$ and the $810 \mathrm{GHz}$ atmospheric windows. The development is a collaboration with SRON (providing the $810 \mathrm{GHz}$ mixer) and the University of Cologne.

The design allows for parallel observations in both channels, which was crucial for the success of the commissioning. To characterize the telescope it was important to operate at the highest possible frequency at which routine operations can be performed (means in the $810 \mathrm{GHz}$ window), while the lower frequency channel was mandatory for pointing and focus references.

Scientifically attractive is the possibility of observing both the mid- $J$ ( $J=4-3$ and $J=7-6$ ) rotational transitions of warm carbon monoxide or both the fine-structure lines ${ }^{3} \mathrm{P}_{1}-{ }^{3} \mathrm{P}_{0}$ and ${ }^{3} \mathrm{P}_{2}-{ }^{3} \mathrm{P}_{1}$ of atomic carbon simultaneously, thus providing good relative calibration.

${ }^{1}$ APEX is a collaboration between the Max-Planck-Institut für Radioastronomie, the European Southern Observatory, and the Onsala Space Observatory.

\section{The FLASH receiver}

The receiver is designed for operation from the left Nasmyth cabin of the APEX-telescope at one of the two PI-receiver mounts near the facility system. To keep the development time short, we adapted the design of the cryostat of the SMART array receiver of the University of Cologne (Graf et al. 2002) to the needs of a dual-channel receiver for APEX. While mechanically compact, the design of the cryostat maintains high flexibility for later system upgrades. The cooling machine, connectors, and all optical components are mounted directly on the dewar top-plate. Because the cold optics also is rigidly connected to this plate, it serves for FLASH as the common reference for the receiver mount and also for the local oscillator (LO) injection optics. This makes for easy maintenance: during three maintenance operations, when dismounting and re-installation of the receiver was required, the system was re-positioned with high precision, introducing a pointing offset of less than $10^{\prime \prime}$.

Cooling is done by a commercial Sumitomo 2-stage closedcycle cooler (SRDK 408D with additional Helium buffer for temperature stabilization at $4 \mathrm{~K}$, with the water-cooled compressor W71D), providing $\approx 1 \mathrm{~W}$ cooling capacity at the $4 \mathrm{~K}$ stage. After only $\approx 8 \mathrm{~h}$ of cool-down time the mixers can be operated at $4.2 \mathrm{~K}$. This makes maintenance work possible within $24 \mathrm{~h}$ of system downtime. The system operates very reliably, and more than 3 months of continuous operation have been easily achieved during the past years.

Tuning of the receiver is made straightforward by adjusting the micrometer screws of the LO-chains and by optimizing the 


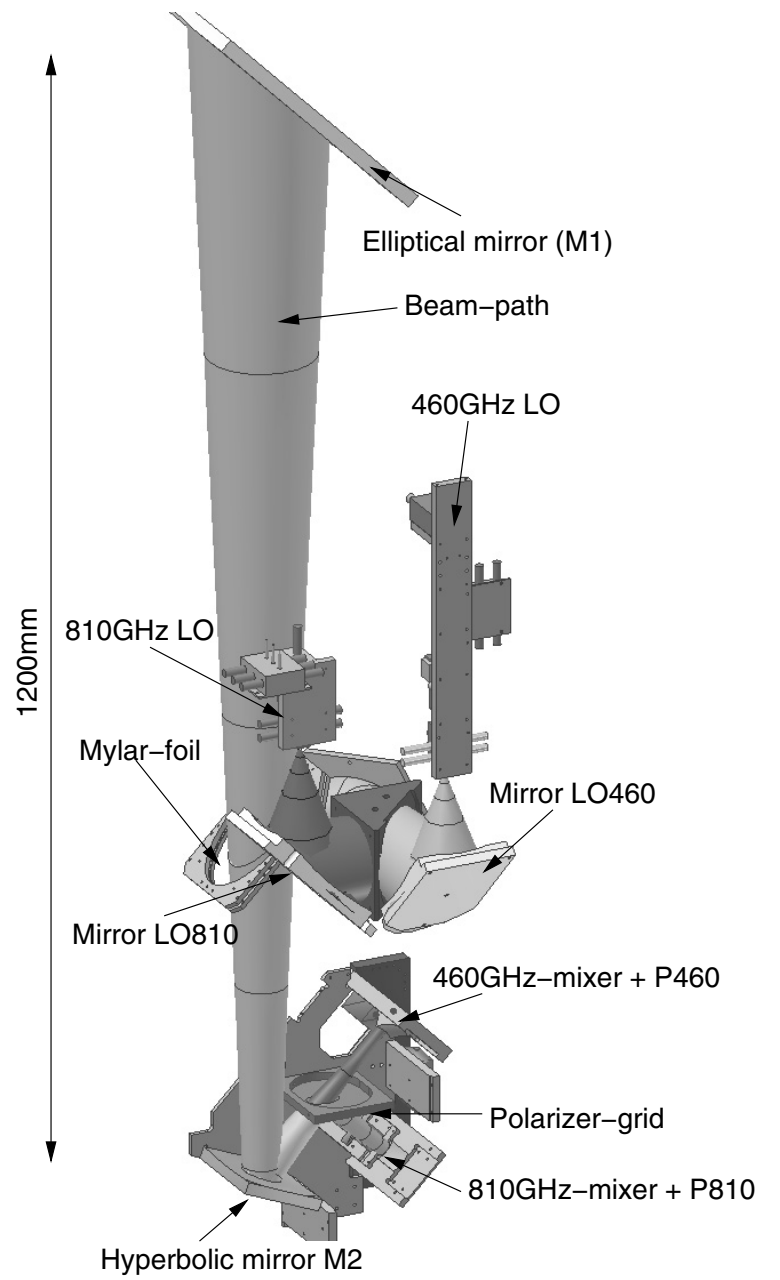

Fig. 1. Schematics of the FLASH optics. The telescope signal is reimaged by the mirrors M1 and M2, forming a Gaussian telescope. Between these mirrors, it is combined with the LO-signals. The signal is then split by polarization and finally matched by individual parabolic mirrors to the mixer-horn antennas (see also Fig. 2).

bias point and the magnet current of the mixers. Remote tuning could be introduced in future.

\subsection{Optics layout}

For a dual-frequency receiver, the main optical requirement is to optimally match both frequency channels, simultaneously, to the telescope. The optical interface to the telescope in the Nasmyth cabin provides a nearly frequency-independent waist location, with the beam size scaling linearly with frequency. The common optics of both FLASH channels follow a Gaussian telescope setup (Goldsmith 1998), which provides both linear scaling of the beam size and frequency-independent waist positions.

The optical layout is explained in Fig. 1: seen from the telescope, inside the Nasmyth cabin the signal first passes a flat pick-up mirror that selects between the three receiver ports in the Nasmyth-A cabin and is then focused into the dewar by the first active mirror of the Gaussian telescope. Inside the dewar, a concave hyperbolic mirror reflects the signal towards a polarizer grid that separates between the two channels. Matching to the individual horn antennas is provided by the parabolic mirrors located directly in front of the mixer blocks (see Table 1). The frequency independence of the setup was first verified by

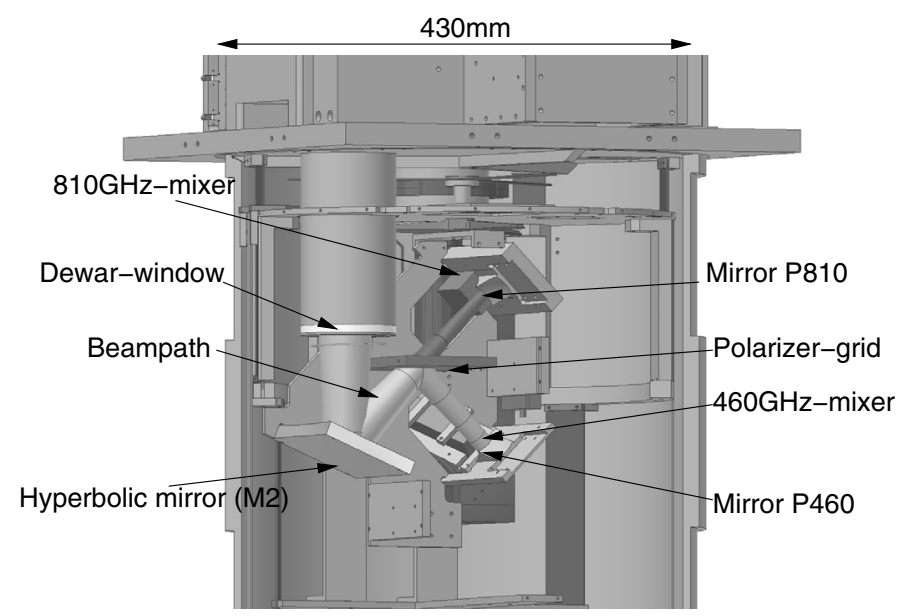

Fig. 2. Picture of the inside of the FLASH dewar. The beam diameter decreases towards the hyperbolic mirror M2. To minimize the window diameter the dewar window has been mounted on a tube that brings it closer to this mirror.

Table 1. Definition of the active optical elements in FLASH ( $f$ is the focal length, $e$ the eccentricity, and $c$ the linear eccentricity of the individual mirror).

\begin{tabular}{llrrr}
\hline \hline Name & Type & $f[\mathrm{~mm}]$ & $e$ & $c[\mathrm{~mm}]$ \\
\hline M1 & elliptic & 833.0 & 0.71 & 1190.4 \\
M2 & hyperbolic & 383.0 & 2.0 & 435.9 \\
P460 & parabolic & 20.74 & 1.0 & $\infty$ \\
P810 & parabolic & 17.29 & 1.0 & $\infty$ \\
\hline
\end{tabular}

numerical simulations, which since then have been confirmed by the uniform radial focus positions of both channels.

The LO signal required for the heterodyne mixing process is provided by two Gunn-diode driven multiplier chains. These chains with their guiding optics to match the LO beams to the mixers are mounted directly on top of the dewar. Both LO-signals are first re-imaged by an elliptical mirror, then optically overlaid by a polarizer grid and finally combined with the sky signal by using a $16 \mu \mathrm{m}$ Mylar foil. Due to the high LO output power available in both frequency bands, there was no need to implement an interferometric diplexer system.

The high machining accuracy of modern CNC-milling machines allowed us to design the cold optics as a monolithic block without any adjustment possibilities. Proper alignment of the receiver to the subreflector is ensured by tilting the whole unit and by tilting the mirror M1 located on top of the receiver within a distance of $\approx 800 \mathrm{~mm}$. A reference laser that has been carefully co-aligned to the telescope elevation axis is used for prealignment during the installation. Not visible in the figures is that a rotary mirror (located in front of the elevation tube) guides the receiver beam to the facility's calibration unit (thus selecting between the hot - ambient temperature - and cold - $73 \mathrm{~K}$ - load, and of course, the sky position).

\subsection{Electrical design}

The electronics consists mainly of three parts, the mixer control, the LO-control and the Intermediate Frequency (IF) electronics. The mixer control electronics was adapted from the CHAMP array (Güsten et al. 1998), providing a stable bias circuit (most critical for reliable operation) and a failure protection circuit for the mixers, a magnet current for suppressing the Josephson 
Table 2. Bandwidth and resolution of both FLASH receiver channels, as well as the beam width on the sky and the main beam efficiency $\eta_{\mathrm{mb}}$ of the telescope (Güsten et al. 2006).

\begin{tabular}{lrr}
\hline \hline FLASH band & $v=460 \mathrm{GHz}$ & $v=810 \mathrm{GHz}$ \\
\hline IF-bandwidth & $2-4 \mathrm{GHz}$ & $4-8 \mathrm{GHz}$ \\
FFTS backends & & \\
max. bandwidth & $2 \times 1 \mathrm{GHz}$ & $2 \times 1 \mathrm{GHz}$ \\
& $2 \times 650 \mathrm{~km} \mathrm{~s}^{-1}$ & $2 \times 370 \mathrm{~km} \mathrm{~s}^{-1}$ \\
channel spacing & $61 \mathrm{kHz}$ & $61 \mathrm{kHz}$ \\
& $0.040 \mathrm{~km} \mathrm{~s}^{-1}$ & $0.022 \mathrm{~km} \mathrm{~s}^{-1}$ \\
beam width $\Theta_{\mathrm{mb}}$ & $13.2^{\prime \prime}$ & $7.6^{\prime \prime}$ \\
main beam efficiency $\eta_{\mathrm{mb}}$ & $60 \%$ & $45 \%$ \\
\hline
\end{tabular}

current at the superconductive mixer junction, and a heater current to remove trapped magnetic flux quanta from the device if necessary. The LO control electronics contains the phase-lock loop circuit to stabilize the LO-frequency, as well as the tunable bias supplies for the multiplier diodes of each of the two LOchains.

The $810 \mathrm{GHz}$ mixer provides an output IF of $4-8 \mathrm{GHz}$, while the $460 \mathrm{GHz}$ mixer operates at 2-4 GHz. The output signals of both mixers are amplified by approx. $60 \mathrm{~dB}$ by cryogenic HEMT (operated at $4 \mathrm{~K}$ for noise reduction) and ambient temperature amplifiers. Because the facility IF system operates at 4-8 GHz, the 2-4 GHz IF of the $460 \mathrm{GHz}$ channel is shifted to 5-7 GHz. The FLASH IF system also includes total-power detectors and adjustable attenuators for both channels. For the first 20 months of operation FLASH was used as a stand-alone system with its own IF-processor and backends. Since March 2006, the IF-processing has been performed by the APEX common IF, which allows us to position the two $1 \mathrm{GHz}$-bands of the novel fast-Fourier transform spectrometers (Klein et al. 2006) freely within the IF band. The minimum channel-spacing selectable is $61 \mathrm{kHz}$ (see also Table 2).

\section{Receiver performance}

The tuning range (see Fig. 3) of the $460 \mathrm{GHz}$-channel (mixer taken from the former CHAMP array) is $\approx 420-500 \mathrm{GHz}$ with a usable IF-bandwidth of $2 \mathrm{GHz}$ (see Table 2). The $810 \mathrm{GHz}-$ channel has a tuning range of $780-880 \mathrm{GHz}$, limited by the LO. The mixer was developed for the Herschel/HIFI program (Jackson et al. 2006; de Lange et al. 2004) and has a much wider tuning range from 760-950 GHz. The intrinsic IF-bandwidth of the mixer is $4-8 \mathrm{GHz}$, out of which $2 \mathrm{GHz}\left(740 \mathrm{~km} \mathrm{~s}^{-1}\right)$ can actually be used with the currently available facility backends.

Both mixers are fixed-tuned DSB (double-side band) SIS mixers. Within the calibration accuracy of the measurements taken with FLASH, as well as from laboratory data of the $810 \mathrm{GHz}$ mixer, the sideband gain ratios are close to 1 for both channels at the mostly used frequencies (CO-lines and CI-lines).

The stability of the receiver, as measured in the telescope environment, is very good: spectroscopic Allan-minimum times (Schieder et al. 1986, see Fig. 4) of more than $300 \mathrm{~s}$ with $61 \mathrm{kHz}$ channel spacing (corresponds to $\approx 75 \mathrm{~s}$ with $1 \mathrm{MHz}$ resolution) allow for efficient on-the-fly observations.

\section{FLASH on sky}

The pointing and focus measurements during the telescope commissioning show that the optics of the receiver performs as designed. The FWHM beam sizes of both channels (see Table 2)

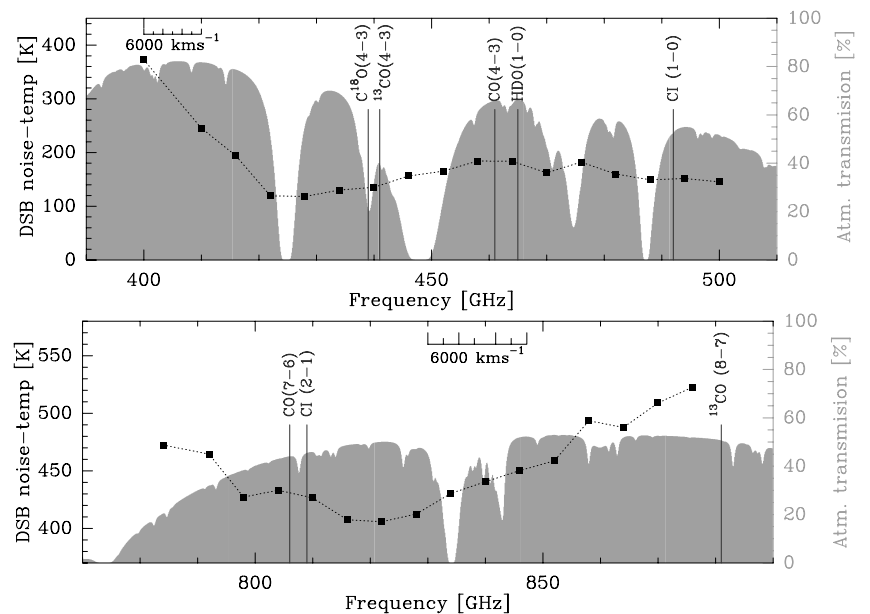

Fig. 3. Tuning range of both FLASH channels superimposed on the atmospheric transmission calculated for good observing conditions with $0.5 \mathrm{~mm}$ precipitable water. The black dots are DSB receiver noise temperatures measured with the facility calibration unit at the telescope. The tuning range of the $460 \mathrm{GHz}$ channel is restricted by the mixer performance at the lower frequency and by the $\mathrm{LO}$ at the upper end of the tuning range. The operating range of the $810 \mathrm{GHz}$ channel is only limited by the tuning range of the LO. For reference, selected frequencies of astrophysical relevant atomic or molecular transitions are indicated.

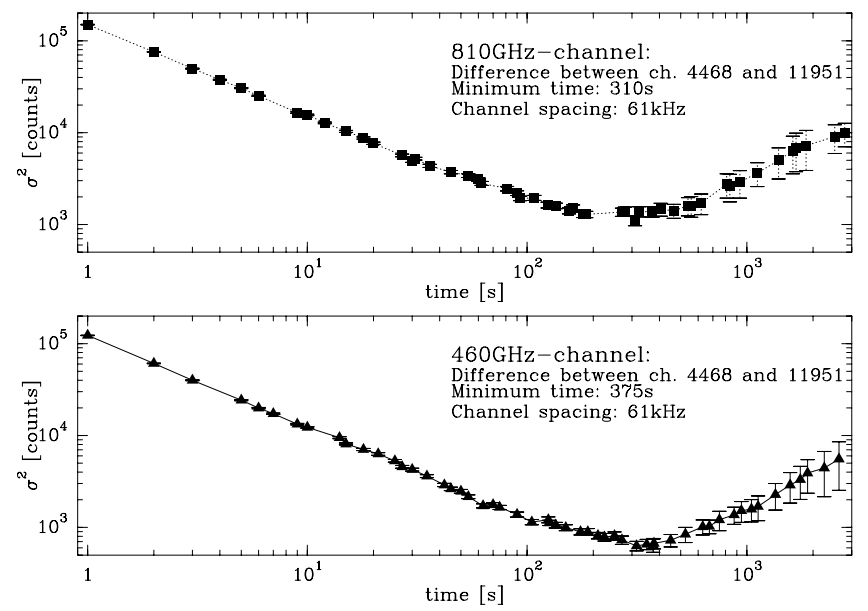

Fig. 4. The spectroscopic Allan-variance plot (using the difference between two spectrometer channels at $\approx 450 \mathrm{MHz}$ distance) of both receiver-channels (terminated at the hot-load during the measurements) shows a minimum time of $>300 \mathrm{~s}$ with a channel spacing of $61 \mathrm{kHz}$. To compare various Allan times, keep in mind that times vary with the square root of the resolution.

correspond well to the diffraction limit of the telescope (7.7" at $810 \mathrm{GHz}$ and $13.6^{\prime \prime}$ at $460 \mathrm{GHz}$ ). The first error beam was determined to be at $-30 \mathrm{~dB}$ for $464 \mathrm{GHz}$ (Güsten et al. 2006) and the width of the error-beam corresponds to the size of the individual telescope panel. The request to operate both channels simultaneously for technical and scientific purposes defines stringent requirements on the receiver (co)alignment, which must be small compared to the beam width of the higher frequency channel and below positional uncertainties introduced by the telescope's pointing accuracy (of 2-3" rms, Güsten et al. 2006). The pointing offset between the two channels was measured to be below 1" (see Fig. 5), which fully complies with these requirements. The radial focus positions were determined to be nearly identical (the difference in $z$-focus is $-9 \mu \mathrm{m}$ only, which is negligible at these wavelengths). 


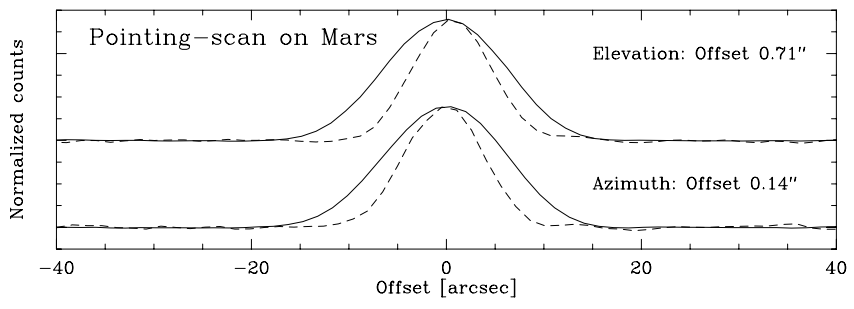

Fig. 5. Total power pointing scan on Mars in elevation (upper plot) and azimuth. The intensities are normalized to visualize the small pointing offset between the two receivers. The data taken at $465 \mathrm{GHz}$ are shown as a solid line.

Galactic Centre CND in $\mathrm{CO}(4-3)$ and $\mathrm{CO}(7-6)$ with APEX/FLASH/FFTS

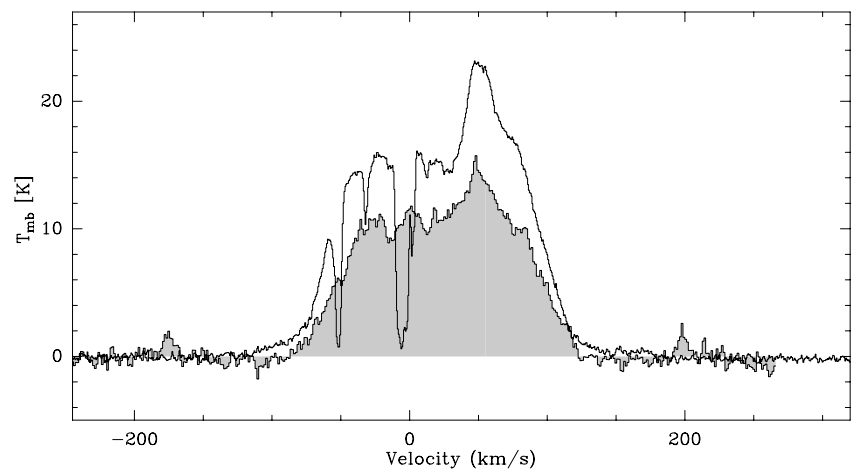

Fig. 6. Spectra towards the galactic centre measured simultaneously in $\mathrm{CO}(4-3)$ and $\mathrm{CO}(7-6)$ with a total integration time of $34 \mathrm{~min}$. The data was calibrated, and only a zero-order baseline was removed. For the velocity range of $\approx 550 \mathrm{~km} \mathrm{~s}^{-1}$ at $806 \mathrm{GHz}$, two $1 \mathrm{GHz}$ FFTS-channels with an overlap range of $150 \mathrm{MHz}$ were used. Outside the source emission baselines are flat.

\section{Conclusions}

With FLASH a dual-frequency state-of-the-art heterodyne receiver has been developed that was essential for the commissioning of the APEX. Since science operation was launched in 2005, the instrument operates as the submillimeter workhorse, and numerous scientific results have been obtained (see the science articles within this special A\&A volume). The PI instrument is available to the APEX user community on a collaborative basis with the MPIfR.

The flexible design of the receiver layout will allow future upgrades to new technologies like the integration of photonic local oscillators, the implementation of new frequency bands (up to $1.5 \mathrm{THz}$ ), and also the possibility of a compact detector array $(2 \times 2$ pixels in 2 polarizations each).

\section{References}

Goldsmith, P. 1998, Quasioptical Systems (IEEE Press)

Graf, U., Heyminck, S., Michael, E., et al. 2002, in Millimeter and Submillimeter Detectors for Astronomy, ed. T. G. Phillips, \& J. Zmuidzinas, Proc. of SPIE, 4855 ,

Güsten, R., Nyman, L. Å., Schilke, P., et al. 2006, A\&A, 454, L13

Güsten, R., Ediss, G., Gueth, F., et al. 1998, in Advanced Technology MMW, Radio, and Terahertz Telescopes, ed. T. G. Phillips, Proc. of SPIE, 3357

Jackson, B. D., de Lange, G., Zijlstra, T., et al. 2006, IEEE Trans. on Microwave Theory and Techniques, 54

Klein, B. et al. 2006, A\&A, 454, L29

de Lange, G., Jackson, B., Eggens, M., et al. 2004, in Astronomical Structures and Mechanisms Technology, ed. J. Antebi, \& D. Lemke, Proc. of the SPIE, 5498

Schieder, R., Rau, G., \& Vowinkel, B. 1986, in Instrumentation for submillimeter spectroscopy, ed. E. Kollberg, Proc. of the SPIE, 598 